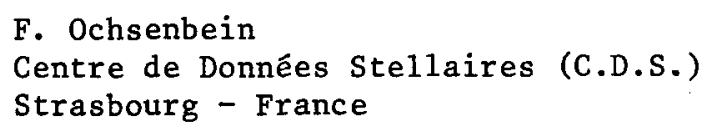

\title{
INTRODUCTION
}

The Bibliographical Star Index (B.S.I.) ries to solve the problem of retrieving the observations or any kind of notes about a star. This file was initiated by Prof. R. Cayrel in 1964 and is going on through a collaboration between Paris-Meudon Observatories and the Stellar Data Center (see Ochsenbein and Spite, 1977). In pratice, the astrophysica1 papers pub1ished on stars are read, the names of all the stars cited in each paper are recorded : simultaneously, the complete bibliographical reference is recorded : name of the author(s), journal, volume, page, year of publication and the full title. These data are keypunched and stored in the computer, each star name is then tested; frequently replaced by a more used designation, and entered into the B.S.I. The retrieval program than lists all the papers with authors, references and full title in which a given star is cited.

\section{DIFFICULTIES AND LIMITATIONS}

Many difficulties prevent from reaching the desired completeness of this bibliographical survey.

1.1. The file begins in the year 1950 ; we do not feel, at the present time, that an extension to earlier publications is a strong need.

1.2. Only 12 journals were covered for the years 1950-1972, and about 40 have been covered since, which represent likely 90 or $95 \%$ of the astronomical literature.

1.3. Lists of more than 1000 objects are excluded; 35 such 1 ists were found for the period 1950-1978, but it should be noted that the majority of these lists are included in catalogs available on magnetic tape or microfiche at the Stellar Data Center.

1.4. The inextricable system in use for stellar designations (see e.g. 171

C. Jaschek and W. Heintz (eds.), Automated Data Retrieval in Astronomy, 171-173.

Copyright $\odot 1982$ by D. Reidel Publishing Company. 
Jaschek et a1. 1980) is likely the main difficulty in this enterprise. At the present time, about 200 catalogs with their numbering system are recorded and the growth ate is about 30 new catalogs used for stellar désignations per year. A software able to handle this large diversity of stellar designations was created, and the use of the Catalogue of Stellar Identifications (see e.g. Ochsenbein et al, 1981) improves the completeness of the B.S.I., at least for the stars brigther than the $10^{\text {th }}$ magnitude. For the fainter stars, we hope that the situation will be much improved in the near future, with the extension of the C.S.I. to fainter stars which has been undertaken by M. Bischoff.

1.5. In fact, the B.S.I. is not only incomplete in several ways, but it is not error-free. As far as we may state some general conclusions about the discovered errors, our experience is that keypunching errors are very rare compared to other sources of errors - and the confusing system used in stellar designations is likely the major source of errors. We thank here the users who have been kind enough to send us lists of corrections, and we encourage all users to do likewise. Remarks, criticisms and suggestions are welcome.

\section{STATISTICAL SUMMARY OF THE B.S.I.}

We summarize in Fig. 1 the contents of the B.S.I. for the period 19501978 : an amount of 12000 papers includes 350000 citations about individual stars, referring to 89000 different stars. This results in a mean number of 4 citations per star for this period, but this mean value includes of $61 \%$ stars quoted only once; Fig. 2 is an histogram of the number of citations per star up to 30 citations ; 1662 stars with more than 30 citations are not plotted in this figure.

Another precision is the part of faint stars in the B.S.I. : roughly $50 \%$ of the stars are fainter than the HD limiting magnitude but in $1978 \mathrm{HD}$ stars still represent $71 \%$ of the citations ; bright stars are obviously more frequently studied than fainter objects.

\section{AVAILABILITE OF THE B.S.I.}

Several facilities have been developed to access the B.S.I. :

a) 3 sets of microfiches covering the periods $1950 / 1979,1973 / 1977$ and 1978 are available at the C.D.S.

b) Bibliography about a list of stars is sent on request (print-out form)

c) The B.S.I. can now accessed through TRANSPAC or EURONET networks in an interactive made.

We hope that this last facility will be frequently used in the future - 


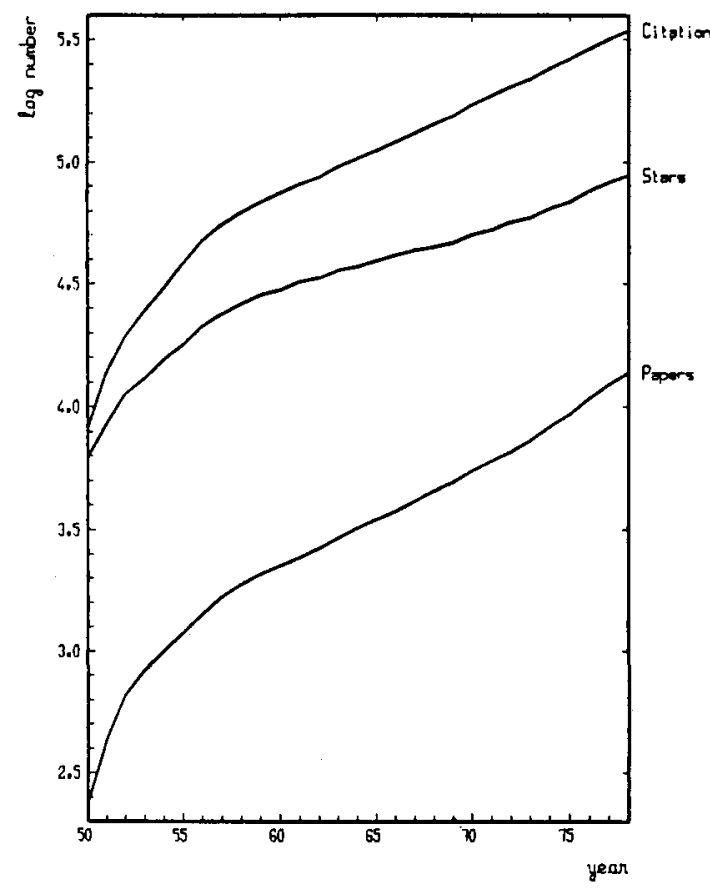

Figure 1

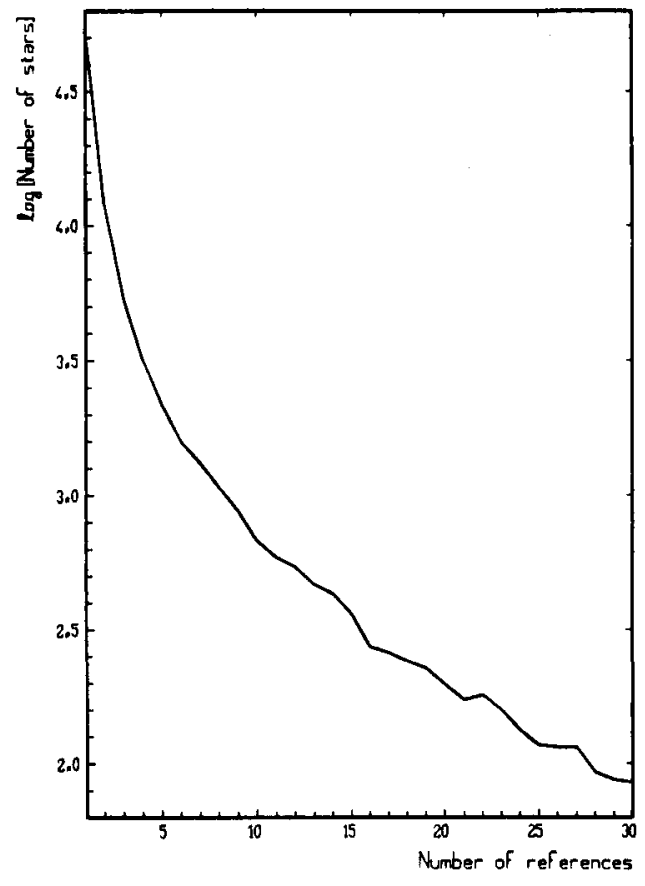

Figure.?

the astronomer gets immediately the more wanted information, and this method implies a reduced amount of work for the staff of the C.D.S.

\section{ACKNOWLEDGEMENT}

It is a pleasure to thank the people who are involved in this rather tedious work : M. Bischoff, R. Cayrel, C. Jaschek, S. Kirchner, R. Lahmek, F. Spite, M.J. Wagner and A. Acker and J. Marcout for the part of the B.S.I. dealing with planetary nebulae (Acker et al, 1981).

\section{REFERENCES}

Acker A., Marcout J., Ochsenbein F. : 1981, Astron. Astrophys. Supp. 43,265

Jaschek C., Ochsenbein F., Bidelman W.P. : 1980, C.D.S. Inf. Bu11. 18, 41

Ochsenbein F., Spite F. : 1977, I.A.U. Co11. 35, Astrophys. Space Sci. Library, 64, 175

Ochsenbein F., Bischoff M., Egret D. : 1981, Astron. Astrophys. Supp. 43,259 